(2) Open Access Full Text Article

\title{
Assessment and monitoring of patients receiving chemotherapy for multiple myeloma: strategies to improve outcomes
}

This article was published in the following Dove Press journal:

Blood and Lymphatic Cancer:Targets and Therapy

24 May 2016

Number of times this article has been viewed

\section{Beth Faiman \\ Jason Valent}

Department of Hematologic Oncology and Blood Disorders, Cleveland Clinic Taussig Cancer Institute, Cleveland, OH, USA
Correspondence: Beth Faiman

Cleveland Clinic Taussig Cancer Institute, 9500 Euclid Avenue R35, Cleveland, OH 44195, USA

Tel + I 2166360200

Email faimanb@ccf.org
$\mathrm{Fax}+\mathrm{I} 2166361707$

\begin{abstract}
Improved understanding as to the biology of multiple myeloma (MM) and the bone marrow microenvironment has led to the development of new drugs to treat MM. This explosion of new and highly effective drugs has led to dramatic advances in the management of MM and underscores the need for supportive care. Impressive and deep response rates to chemotherapy, monoclonal antibodies, and small molecule drugs provide hope of a cure or prolonged remission for the majority of individuals. For most patients, long-term, continuous therapy is often required to suppress the malignant plasma cell clone, thus requiring clinicians to become more astute in assessment, monitoring, and intervention of side effects as well as monitoring response to therapy. Appropriate diagnosis and monitoring strategies are essential to ensure that patients receive the appropriate chemotherapy and supportive therapy at relapse, and that side effects are appropriately managed to allow for continued therapy and adherence to the regimen. Multiple drugs with complex regimens are currently available with varying side effect profiles. Knowledge of the drugs used to treat MM and the common adverse events will allow for preventative strategies to mitigate adverse events and prompt intervention. The purpose of this paper is to review updates in the diagnosis and management of MM, and to provide strategies for assessment and monitoring of patients receiving chemotherapy for MM.
\end{abstract}

Keywords: multiple myeloma, treatment, symptoms, assessment, monitoring, symptom management, targeted therapies

\section{Introduction}

Multiple myeloma (MM) is an incurable, but highly treatable cancer characterized by an overproliferation of bone marrow plasma cells, which leads to the production of a monoclonal protein. Through a series of genetic changes, genetic mutations, and cellular alterations, the normal plasma cell turns malignant. Cancerous plasma cells overproduce clonal immunoglobulin (Ig) proteins which cause organ destruction. Although the genetic makeup of the tumor itself and patient symptoms at presentation are heterogeneous, common signs and symptoms of MM exist at diagnosis. Known as "CRAB" criteria, the pneumonic stands for hyperCalcemia, Renal insufficiency, Anemia, and Bone damage. The incidence of these at diagnosis is as follows: anemia $73 \%$, bone pain $68 \%$, renal insufficiency $19 \%$, and hypercalcemia $13 \%$. ${ }^{1}$ To delay worsening of existing organ damage, or to prevent future organ damage, prompt treatment of the malignant plasma cells with chemotherapy is warranted.

As of 2012, it is estimated that 65,000 individuals are living with MM globally and comprise $\sim 2 \%$ of all cancer types. ${ }^{2}$ The incidence of MM is expected to increase over the next decade. The etiology of MM is unknown, but the risk is associated with 
increasing age, obesity, and race. $\mathrm{MM}$ is more prevalent in individuals over the age of 65 and is nearly two times higher in African-American individuals and men. Obesity and high body mass index further increases the risk to develop monoclonal gammopathy of undetermined significance (MGUS) and potential $\mathrm{MM}^{3}$

\section{Initial evaluation}

All patients who present with an abnormal paraprotein should undergo a thorough laboratory, radiologic, and physical assessment, as multiple factors are considered to make the correct diagnosis. In addition to usual tests such as a complete blood count with differential count and complete metabolic panel testing, common biomarkers to assess MM disease include serum protein electrophoresis with quantification of monoclonal protein, urine protein electrophoresis, and kappa/lambda serum free light chain (FLC). Beta-2 microglobulin and lactate dehydrogenase (LDH) also assess the burden of disease. ${ }^{4}$

Genetic changes and aberrations to the plasma cell clone and within the bone marrow microenvironment can lead to myeloma cell development and breed resistant disease over time. Three main ways to identify genetic aberrations in MM patients on clonal plasma cells include: 1) karyotype chromosome analysis, 2) fluorescence in situ hybridization, and 3) gene expression profiling.,6 Traditional MM-specific tests (such as serum protein electrophoresis, urine protein electrophoresis, kappa/lambda FLCs, and LDH) are combined with these methods to estimate prognosis. Clinicians should have knowledge of biomarker and genomic results which are essential when stratifying patients into appropriate risk categories, as the results guide treatment selection. ${ }^{7-10}$

In addition to laboratory testing and bone marrow evaluation with biopsy, radiologic testing at diagnosis is important to determine if bone disease is present. Back or bone pain is a common presenting symptom of MM, as destructive, painful osteolytic lesions are common at diagnosis. Pain can be a result of increased osteoclastic activity, skeletal fractures, bone marrow plasmacytosis, or can be due to a plasmacytoma. ${ }^{11}$ Not all lesions are painful; however, assessment of MM bone disease is critical to delineate one's diagnosis and should continue periodically with disease monitoring. At baseline, conventional plain radiography with a metastatic skeletal survey should be performed and include the axial skeleton, skull, and large cortical bones. An abnormal survey will reveal osteolytic lesions, osteopenia, or fractures in $\sim 80 \%$ of patients with MM at diagnosis. However, $\sim 30 \%$ bone loss should have occurred before bone lesions are detected. ${ }^{12-14}$
For patients with a high suspicion of extramedullary disease or occult bone disease not seen on conventional radiology, 18-F-fluorodeoxyglucose positron emission tomographycomputed tomography, positron emission tomographymagnetic resonance imaging (MRI), or MRI of spine or suspicious lesions should be considered. ${ }^{15}$

\section{Diagnosis and differential diagnosis of MM}

The diagnosis of MM continues to evolve as new drugs that effectively treat MM are available. In previous years, the diagnosis of MM was made if patients had experienced a myeloma-defining event, or "CRAB" criteria were achieved. MM occurs along a spectrum of plasma cell disorders that range from an asymptomatic and often benign condition called MGUS, smoldering MM (SMM), and symptomatic MM. It is currently recommended that patients with MGUS or SMM be only treated within the context of a well-designed clinical trial. Currently, only symptomatic MM requires immediate treatment (Table 1). Thus, it is essential that an accurate diagnosis of MM be confirmed prior to initiating plasma cell directed therapy. ${ }^{4,16}$

MGUS is present in $\sim 3 \%-4 \%$ of the US population in individuals over the age of 50 years and often precedes the diagnosis of MM and other plasma cell disorders. ${ }^{1,17}$ Patients qualify for an MGUS diagnosis if they lack CRAB criteria and have no evidence of organ damage related to the monoclonal protein. ${ }^{9}$ There are three types of paraproteinemia: IgG, IgA, and IgM. Individuals with non-IgM MGUS carry a relatively low risk of progression to $\mathrm{MM}$, lymphoma, amyloidosis, or other plasma cell dyscrasias at a rate of $<1 \%$ per year. One's individual risk of progression, however, is based on the level of monoclonal protein concentration in the blood or urine, serum FLC burden, and percentage of bone marrow plasmacytosis. ${ }^{16,18-20}$ Patients with SMM have a higher burden of disease, but remain without $\mathrm{CRAB}$ criteria or evidence of organ damage. While some patients with SMM behave more like MGUS, meaning a low risk of progression and quiescent disease course, a majority of patients will progress within 2 years if bone marrow plasma cells are $>10 \%$ and there is elevated serum FLC ratio, and $\mathrm{t}$ (4:14), gain 1q, or other genetic abnormalities are present. ${ }^{21,22}$ It is important to consider that $\sim 7 \%$ of newly diagnosed MGUS or MM patients will have a concurrent diagnosis of amyloidosis. Close attention to renal, cardiac, or other abnormalities should alert the clinician to assess for the presence of amyloid, which would require emergent treatment. 
Table I Diagnostic criteria for multiple myeloma and differential diagnosis

\begin{tabular}{|c|c|c|c|}
\hline Condition & MGUS & SMM & Active multiple myeloma \\
\hline$\%$ of clonal plasma cells in bone marrow & $<10 \%$ & $10 \%-60 \%$ & $\geq 10 \%$ \\
\hline $\begin{array}{l}\text { Myeloma-defining events: hypercalcemia, } \\
\text { anemia }<10 \mathrm{~g} / \mathrm{dL} \text { or } 2 \mathrm{~g} \text { below normal, } \\
\text { renal insufficiency with creatinine }>2 \mathrm{~g} / \mathrm{dL} \text {, } \\
\text { bone disease (more than one focal lesion } \\
\text { on MRI or widespread lytic lesions) }\end{array}$ & None & $\begin{array}{l}\text { None; however, patients are at a high risk } \\
\text { for progression if diffuse abnormalities or } \\
\text { one focal lesion on MRI }\end{array}$ & $\begin{array}{l}\text { Yes, or any one: } \\
\text { I) clonal BMPCs } \geq 60 \% \text {, or } \\
\text { 2) serum FLC ratio } \geq 100 \text { (plus measurable } \\
\text { involved } F L C \text { level } \geq 100 \mathrm{mg} / \mathrm{L} \text { ), or } \\
\text { 3) more than one focal lesion on MRI scan }\end{array}$ \\
\hline Likelihood of progression & $\sim 1 \%$ per year & $\begin{array}{l}\sim 10 \% \text { per year; higher if serum involved/ } \\
\text { uninvolved FLC ratio } \geq 8 \text { (but }<100 \text { ), } \\
\text { IgA isotype and immunoparesis }\end{array}$ & Not applicable \\
\hline Treatment & $\begin{array}{l}\text { No, watchful } \\
\text { waiting }\end{array}$ & $\begin{array}{l}\text { Yes for high risk (especially in the context } \\
\text { of clinical trial) } \\
\text { No for others }\end{array}$ & Yes \\
\hline
\end{tabular}

Note: Data from Durie et al, ${ }^{16}$ Rajkumar et al. ${ }^{4}$

Abbreviations: BMPC, bone marrow plasma cell percentage; FLC, free light chain; IgA, immunoglobulin A; MGUS, monoclonal gammopathy of undetermined significance; MRI, magnetic resonance imaging; SMM, smoldering multiple myeloma.

In 2014, the International Myeloma Working Group revised the criteria for diagnosis of MM to include clonal bone marrow plasma cell percentage $\geq 60 \%$, an involved to uninvolved serum FLC ratio $\geq 100$, and $>1$ focal lesions on MRI. ${ }^{4,14,16}$ The update was based on prior studies which supported an increased risk of progression to MM based on these three aforementioned factors. The presence of greater than one lesion on MRI was associated with a median time to progression of 13 months, and $70 \%$ of individuals had progressed at 2 years. In a study of patients with SMM, those with clonal bone marrow plasma cell percentage of $60 \%$ or greater had all progressed to symptomatic MM within 2 years. ${ }^{23}$ Elevated serum FLC ratios $>100$ and involved FLC $>100 \mathrm{mg} / \mathrm{L}$ have been linked to an increased risk of MM disease progression in as less as 18 months. ${ }^{24-26}$ The recommendation to include the presence of more than one focal lesion was based on a study which evaluated the use of whole-body MRI in MM. The presence of a single lesion on MRI was associated with a median time to progression of 13 months, and $70 \%$ of individuals in the study progressed at 2 years. ${ }^{27}$

Prognosis and risk stratification is based on the revised international staging system (ISS), Durie-Salmon staging system, and genetic classification with chromosomal abnormalities. ${ }^{5,28-32}$ Host factors and genetic factors are two criteria which influence prognosis. Host factors affect prognosis and include increased age and comorbid illnesses. Genetic factors include IgH translocations and high LDH levels. A majority of patients will have trisomy or hyperdiploid MM with $\mathrm{t}$ $(11 ; 14)$ or $\mathrm{t}(6 ; 14)$, which confers a favorable prognosis. Approximately $10 \%$ will have intermediate-risk disease with fluorescent in situ hybridization $t(4 ; 14)$. The remainder of patients are categorized as high risk with $\mathrm{t}(14 ; 16)$, $\mathrm{t}(14 ; 20), 17 \mathrm{p}$ deletion, or a high-risk gene expression profiling signature., 9

\section{Treatment of MM}

Just as the diagnosis of MM continues to evolve, so do its treatment goals. While a cure may be possible for a small percentage of patients, usually those with standard- or low-risk disease, the primary goal of treatment is disease control and improvement of symptoms. Prompt initiation of chemotherapy to prevent worsening of symptoms or organ damage is recommended. Despite the availability of methods to assess the risk status and disease burden, there is no clear consensus as to the best treatment for newly diagnosed MM patients, but it is clear that the newer drugs are superior to older drugs and should be included in treatment.

With four new drug approvals by the US Food and Drug Administration (FDA) in 2015, there are now seven therapeutic classes of drugs approved for use in the US to treat myeloma. Examples include: 1) alkylating agents (melphalan, cyclophosphamide); 2) other chemotherapy (doxorubicin, vincristine, etoposide); 3) corticosteroids (dexamethasone, prednisone); 4) histone deacetylase inhibitors (panobinostat); 5) immunomodulatory agents (lenalidomide, pomalidomide, thalidomide); 6) monoclonal antibodies (daratumumab and elotuzumab [ELO]); and 7) proteasome inhibitors (bortezomib, carfilzomib, and ixazomib).$^{33}$ The survival of patients with MM has increased over the past decade as new classes of drugs with novel mechanisms of action have been added to the treatment armamentarium, although it 
remains unclear which drug or drug combination should be given sequentially. ${ }^{34}$ Most will agree that combination therapies, given at diagnosis or relapse, have provided improved response rates and progression-free survival (PFS), compared to standard chemotherapy alone. ${ }^{35-46}$ Current guidelines suggest every patient with MM should be evaluated for hematopoietic stem cell transplantation at the time of diagnosis. Regardless of transplant eligibility, prolonged doses of melphalan should be avoided in transplant-eligible patients as melphalan can impair the ability to collect stem cells. For most, combination therapy with two drugs or three drugs upfront remains common practice. Table 2 highlights the common side effects of treatment categorized by body system and general recommendations, as the clinicians have to be aware of them for health promotion.

\section{Bortezomib}

Bortezomib is a proteasome inhibitor that has been extensively studied and shown to improve PFS and overall survival (OS) in several studies on newly diagnosed or relapsed MM. ${ }^{35,38,39}$ In a trial of 682 patients with newly diagnosed MM (NDMM) and ineligible for autologous stem cell transplant, each of them was randomly assigned to receive nine 6-week cycles of melphalan $\left(9 \mathrm{mg} / \mathrm{m}^{2}\right)$ and prednisone $\left(60 \mathrm{mg} / \mathrm{m}^{2}\right)$ on days $1-4$. Melphalan and prednisone (MP) was given to patients either alone or in combination with bortezomib $\left(1.3 \mathrm{mg} / \mathrm{m}^{2}\right)$ on days $1,4,8,11,22,25,29$, and 32 for four cycles. This was followed by a maintenance phase for responding patients (cycles 5-9). The combination of bortezomib + MP was superior to MP alone, as 71\% of patients had achieved at least a partial response versus $35 \%$ of patients receiving MP alone. ${ }^{39}$ Side effects of peripheral neuropathy and diarrhea were greater in the bortezomib, melphalan, and prednisone (VMP) versus MP groups, thus close attention to peripheral neuropathy symptoms and diarrhea is warranted.

Bortezomib has been studied in combination with lenalidomide and dexamethasone in newly diagnosed and relapsed MM. ${ }^{47-49}$ Recent results of a large cooperative group study showed a clinically meaningful and statistically significant improvement in PFS among groups. In this Phase III, multicenter Southwest Oncology Group study, bortezomib, lenalidomide, and dexamethasone (VRd) was compared to standard therapy with lenalidomide and dexamethasone ( $\mathrm{Rd})$. The primary end point was PFS. Of 474 patients evaluable for response, median PFS was 43 months (VRd) versus 31 months $(\mathrm{Rd})$. Median OS was not reached in the VRd group versus 63 months in the Rd group. The most common hematologic adverse events (AEs) were low hemoglobin $(\mathrm{VRd}=13 \%$ vs $\mathrm{Rd}=16 \%)$, leukopenia $(\mathrm{VRd}=14 \%$ vs $\mathrm{Rd}=16 \%)$, lymphopenia (VRd $=23 \%$ vs $\mathrm{Rd}=18 \%$ ), neutropenia $(\mathrm{VRd}=19 \%$ vs $\mathrm{Rd}=21 \%)$, and low platelets $(\mathrm{VRd}=$ $18 \%$ vs $\mathrm{Rd}=14 \%$ ). Side effects such as hypokalemia, muscle weakness, diarrhea, and dehydration were more common in the VRd versus Rd groups, although the incidence of thromboembolic events was similar ( VRd $=8 \% \mathrm{vs} \mathrm{Rd}=9 \%$ ). Sensory neuropathy was more common in the VRd versus Rd groups (23\% vs 3\%), with greater severity in the VRd versus $\mathrm{Rd}$ groups $(24 \%$ vs $5 \%$ ). It is important to note, however, that administering bortezomib by the subcutaneous (SC) route will minimize the incidence and severity of peripheral neuropathy. ${ }^{50,51}$ The impressive PFS data in this Southwest Oncology Group study support the use of a threedrug combination upfront; however, the improved survival is counterbalanced by increased risk of neurotoxicity. Thus, for individuals who receive this regimen, zoster prophylaxis, neuropathy surveillance, SC bortezomib administration, dose adjustments, and education are essential to minimize the risk of AEs. Appropriate laboratory and disease monitoring is also recommended. ${ }^{9}$

\section{Lenalidomide}

Lenalidomide and dexamethasone have proved to be superior to dexamethasone alone in randomized clinical trials in patients with newly diagnosed and relapsed MM, and can be given to patients who are transplant eligible and ineligible. ${ }^{52-54}$ In the Frontline Investigation of Revlimid (lenalidomide) and dexamethasone versus Standard Thalidomide study, 1,623 NDMM patients were randomized to one of three treatment arms. These included: 1) standard $\mathrm{Rd}$ in 28-day cycles until disease progression $(n=535), 2) \mathrm{Rd}$ for 72 weeks (18 cycles; $n=541 ; R d 18)$, and 3 ) melphalan, prednisone, and thalidomide (MPT) for 72 weeks $(n=547)$. The median PFS was 25.5 months in the continuous Rd arm, 20.7 months in the Rd18 arm, and 21.2 months in the MPT arm. The 4-year OS rate in the $\mathrm{Rd}$ continuous group was 59\% compared to $51 \%$ among patients who received MPT. ${ }^{45}$ The incidence of grade 3 side effects was lower in the continuous Rd group versus MPT group ( $85 \%$ vs $89 \%$ ), primarily because of the higher incidence of neuropathy in the MPT versus Rd groups ( $9 \%$ vs $1 \%$ respectively).

The $\mathrm{Rd}$ combination is generally well tolerated and effective in transplant-eligible and -ineligible patients. The most common starting dose for patients with a creatinine clearance $>60 \mathrm{~mL} / \mathrm{min} / 1.73 \mathrm{~m}^{2}$ is $25 \mathrm{mg}$ orally on days 1-21 of a 28-day cycle in combination with dexamethasone 


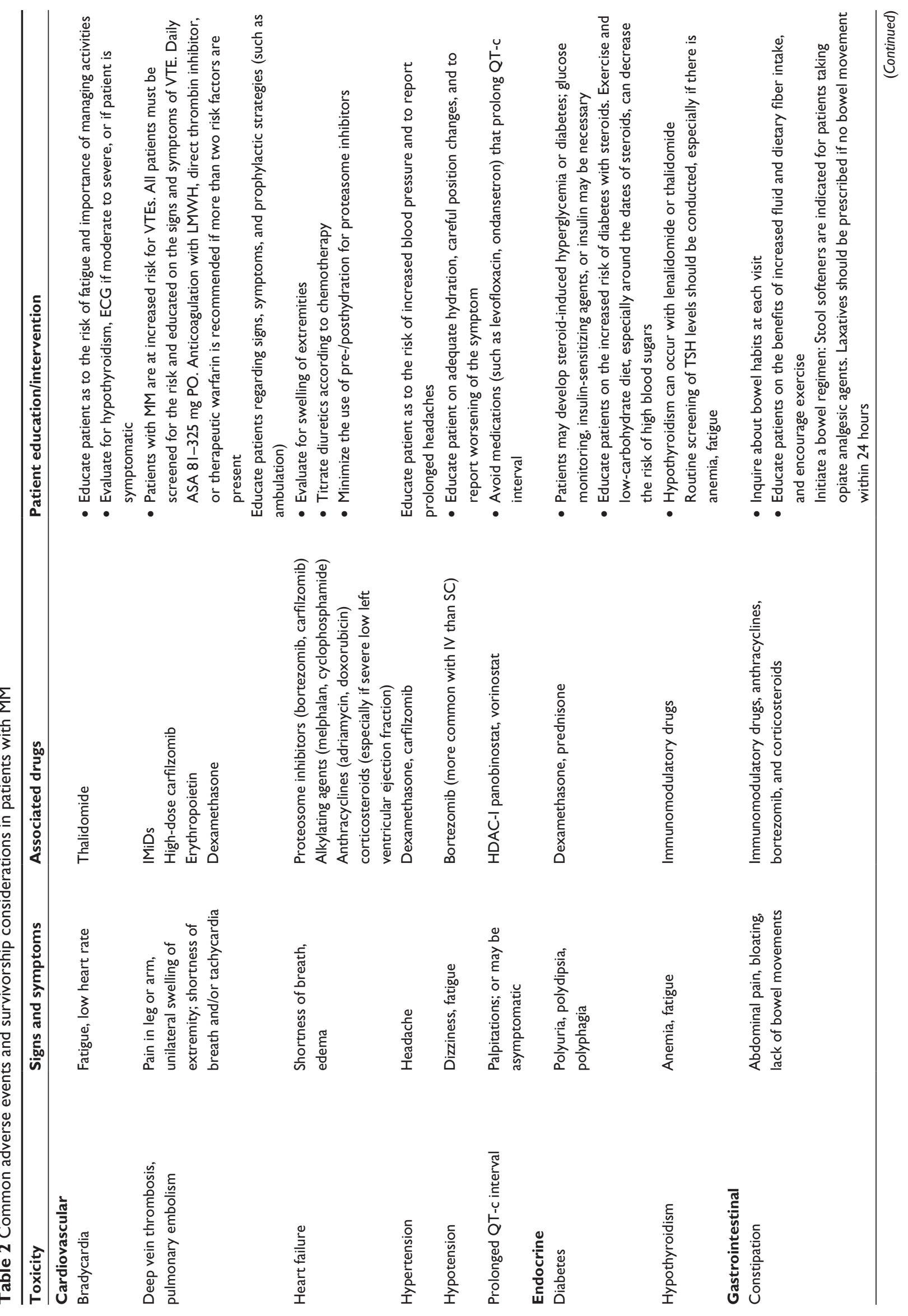




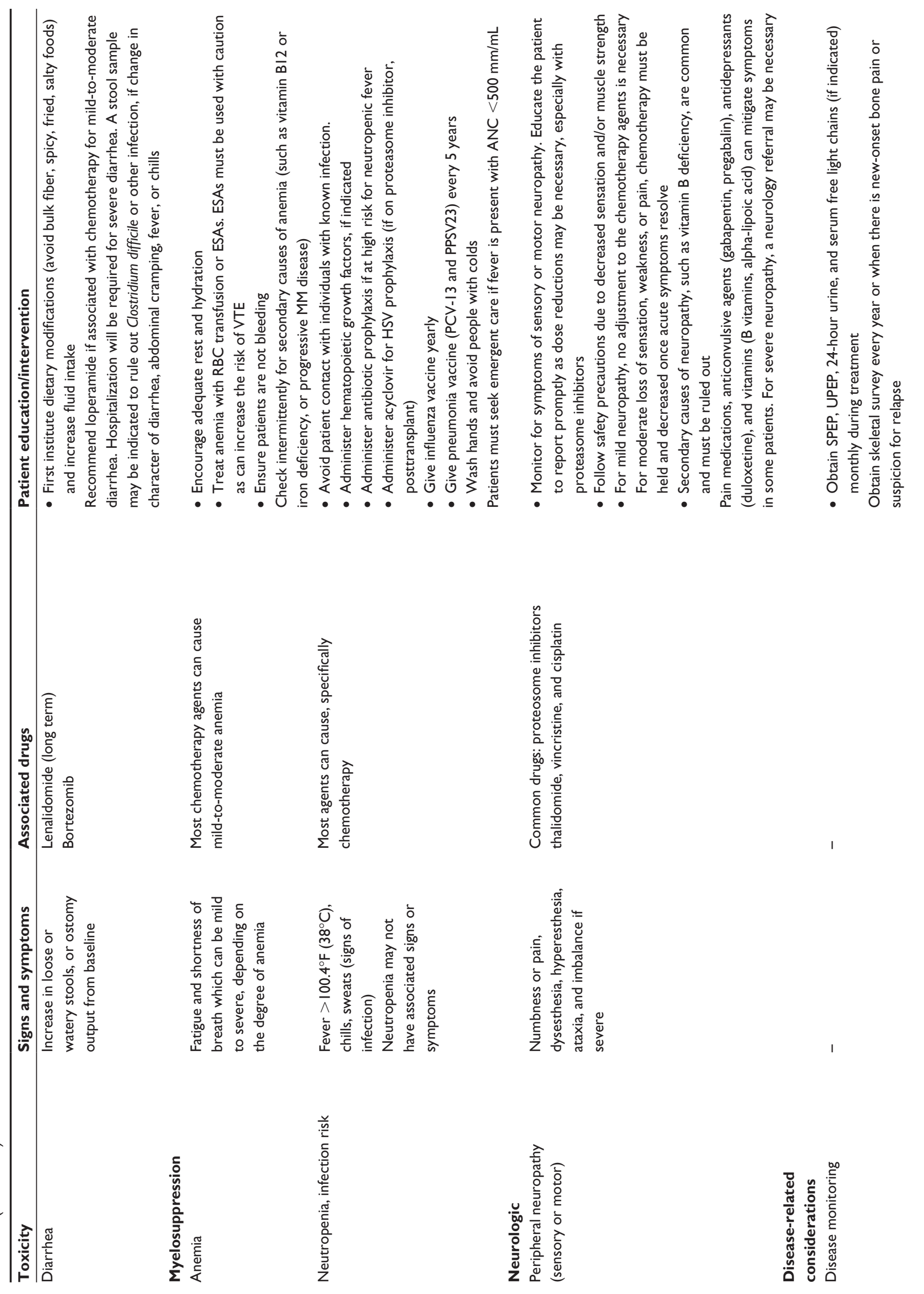



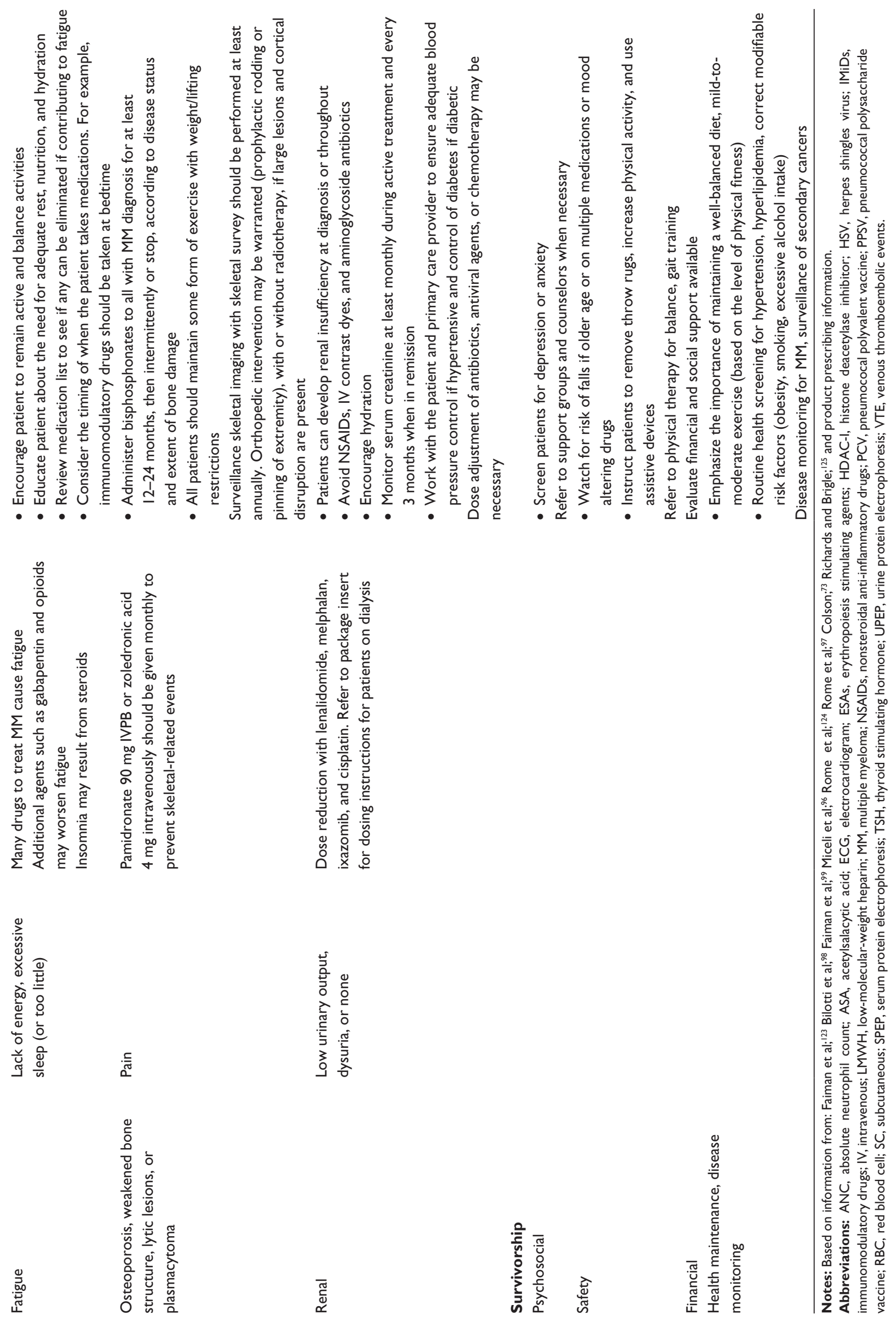
$40 \mathrm{mg}$ orally weekly. However, the dose of lenalidomide and dexamethasone should be carefully considered. Recent studies suggest that patients do better with reduced doses of lenalidomide and dexamethasone, which is especially found in patients of older age $(>75)$ or in those with decreased renal function. ${ }^{55-58}$

\section{Carfilzomib}

Carfilzomib, an epoxyketone proteasome inhibitor, differs from bortezomib in that it binds selectively and irreversibly to the proteasome. ${ }^{44}$ Carfilzomib has demonstrated activity as a single agent and in combination with other drugs. The combination of carfilzomib, lenalidomide, and dexamethasone is approved by the FDA, in combination with $\mathrm{Rd}$, based on the results of a large, Phase III study called the ASPIRE trial. In this trial, 792 patients with relapsed MM were randomized to receive intravenous (IV) carfilzomib + Rd (KRd) or $\mathrm{Rd} .{ }^{44}$ A higher overall response rate (ORR) was observed in the KRd versus Rd groups (87.1\% vs 66.7\%), and was accompanied by a $31 \%$ decrease in the risk of progressive disease (PD) or death. The most common side effect in both groups was myelosuppression. The study supports the use of a three-drug regimen (proteasome inhibitor, immunomodulatory drug, and corticosteroid) to treat relapsed and refractory MM. ${ }^{44}$

In patients with relapsed and refractory MM, carfilzomib is effective when administered IV in doses from 27 to $56 \mathrm{mg} / \mathrm{m}^{2}$ IV twice weekly. ${ }^{59-64}$ In the Phase III ENDEAVOR trial, 929 patients were randomized to receive carfilzomib + dexamethasone $(\mathrm{Kd})$ or bortezomib and dexamethasone (Vd). In the study, treatment with $\mathrm{Kd}$ doubled the PFS, compared to Vd (18.7 months compared with 9.4 months, respectively). ${ }^{59}$ Carfilzomib is also being investigated in combination with cyclophosphamide and dexamethasone in patients with an average age of 74 years, and in doses as high as $56 \mathrm{mg} / \mathrm{m}^{2}{ }^{65-67}$ Additional studies have reported promising results with carfilzomib in combination with pomalidomide. In a trial of patients with relapsed and refractory MM, an ORR of $50 \%$ with carfilzomib and pomalidomide was observed. ${ }^{68}$ Preliminary results of this and other studies demonstrate promising response rates with a tolerable safety profile, no unexpected toxicities, and merit the investigation of higher doses and in combination with other agents. ${ }^{69-71}$

Common hematologic toxicities are thrombocytopenia, lymphopenia, and anemia. Nonhematologic toxicities include hypokalemia, cough, respiratory tract infections, and diarrhea. It is interesting that although venous thromboembolic event (VTE) prophylaxis is generally not recommended for proteasome inhibitors, there was an increase in VTE among patients who received $\mathrm{Kd}$ versus $\mathrm{Vd}$ in the ENDEAVOR trial, and therefore, VTE prophylaxis is recommended. ${ }^{59}$ Since cardiac events such as congestive heart failure and cardiac arrest can occur, echocardiogram and electrocardiogram should be performed at baseline and periodically, as warranted. A longer infusion time of carfilzomib to be given over 30 minutes may also effectively reduce shortness of breath or symptoms and is currently recommended for all doses. ${ }^{72,73}$

\section{Pomalidomide}

Pomalidomide is an oral immunomodulatory agent that was approved for use by the FDA in 2013 for treatment of relapsed and refractory MM based on a Phase II randomized study of pomalidomide alone or in combination with dexamethasone. ${ }^{74}$ An ORR of 33\% was observed in the pomalidomide and dexamethasone group, with median PFS of 4.2 months. The combination was well tolerated in heavily pretreated patients who are refractory to bortezomib and lenalidomide. Neutropenia was mild and the most notable AE in the study. ${ }^{42}$ Results of ongoing studies of pomalidomide in combination with bortezomib, ixazomib, cyclophosphamide, daratumumab, and pembrolizumab will undoubtedly further support the use of pomalidomide in MM, as preliminary results show high response rates in these combination regimens. ${ }^{75-78}$

\section{Panobinostat}

Panobinostat is pan-deacetylase (histone deacetylase) inhibitor that, through epigenetic alterations, impairs the growth and survival of MM cells. This an oral agent which is currently indicated for relapsed and refractory MM based on the results of a Phase II randomized study in which patients received bortezomib, panobinostat, and dexamethasone (PAN-BTZ-Dex) or BTZ-Dex alone. Patients who received PAN-BTZ-Dex experienced a PFS advantage of 10.6 months versus 5.8 months in patients who received BTZ-Dex alone. ${ }^{79}$ A recent analysis showed a PFS benefit of 7.8 months for the PAN-BTZ-Dex group among those who received more than two regimens. ${ }^{80}$ Common AEs in the PAN-BTZ-Dex versus BTZ-Dex group included thrombocytopenia (67\% vs $31 \%)$, diarrhea ( $26 \%$ vs $8 \%$ ), asthenia or fatigue ( $24 \%$ vs $12 \%$ ), and peripheral neuropathy ( $18 \%$ vs $15 \%$ ).

Based on common side effects in the study, patients should be closely monitored for diarrhea, electrolyte imbalance, and peripheral neuropathy. In the study, there was a high incidence of diarrhea, and 25\% was severe. Therefore, loperamide is recommended to ameliorate diarrhea symptoms in patients who receive this three-drug regimen. Also, assessment and correction of blood electrolyte abnormalities is critical to prevent fatigue, muscle cramping, and potential 
electrocardiogram changes. Baseline and ongoing electrolyte monitoring should be employed on at least a monthly basis and include a complete metabolic panel + magnesium. Electrocardiogram monitoring to assess for prolongation of the QT-c interval should be performed at baseline and periodically in all patients and more often in those with known cardiac dysfunction. In the study, a high incidence of neuropathy was reported, likely due to IV administration of bortezomib. Thus, bortezomib should be given SC to minimize the onset of neuropathy, and all patients should be monitored closely for the onset of peripheral neuropathy symptoms. ${ }^{73,79}$

\section{Ixazomib}

Ixazomib is an oral proteasome inhibitor approved in the US for treatment of MM in patients who received one prior therapy. In the TOURMALINE MM-1 study, 722 patients who received one to three prior lines of therapy and were not refractory to prior lenalidomide or proteasome inhibitor based therapy were randomized to receive oral ixazomib $4 \mathrm{mg}$ on days $1,8,15$ every 28 days; oral lenalidomide $25 \mathrm{mg}$ on days 1-21 q 28 days; and dexamethasone $40 \mathrm{mg}$ on days 1 , $8,15,22$ (IRd), or lenalidomide and dexamethasone ( $\mathrm{Rd}$ ) for up to twelve 28-day induction cycles. At interim analysis, an increased median PFS in the IRd versus Rd groups (20.6 vs 14.7 months) without a substantial increase in overall toxicity was observed. ${ }^{81}$ Fairly common but mild side effects in patients who received IRd included peripheral neuropathy, diarrhea, constipation, rash, and myelosuppression. Therefore, education and assessment of these side effects is highly important.

\section{Daratumumab}

Daratumumab is a human, IgG1 kappa monoclonal antibody against CD38 with single agent activity, and is also effective in combination with other therapies. Daratumumab is approved for use in relapsed and refractory MM in patients who have received more than three prior therapies. ${ }^{82,83}$ A recent analysis of two key studies demonstrated exciting results and single agent activity. Among 148 heavily pretreated patients with MM, double-refractory to a proteosome inhibitor and immunomodulatory drug, a group that generally confers a poor prognosis, a combined ORR of $31 \%$ was observed.

Daratumumab is generally well tolerated; yet, there is a high incidence of infusion reactions with daratumumab, particularly with the first dose. Thus, it is recommended to give an IV corticosteroid (methylprednisolone $100 \mathrm{mg}$ or equivalent dose), oral antipyretics (acetaminophen 650-1,000 mg PO), plus an antihistamine (such as diphenhydramine $25-50 \mathrm{mg} \mathrm{PO} / \mathrm{IV}$ or equivalent) prior to each infusion. Postinfusion medications with an oral corticosteroid (such as methylprednisone $20 \mathrm{mg}$ ) should also be given on the first and second days after all infusions, and especially for the first four infusions. ${ }^{84}$ Anecdotal evidence supports the use of montelukast and loratadine the day before daratumumab infusion and for the first and second days after the first three infusions.

Red blood cell (RBC) transfusions are often necessary in this heavily pretreated MM population. In an ongoing study, $40 \%$ of patients receiving daratumumab at a dose of $16 \mathrm{mg} / \mathrm{kg}$ as a monotherapy required blood transfusions. Blood typing for compatibility may be an issue, as daratumumab binds to CD38, a protein that is ubiquitously expressed on myeloma cells, but also expressed, to a lesser extent, on RBCs. As daratumumab interferes with antibody testing by binding to endogenous CD38 on RBCs, resulting in pan-agglutination, blood banks should be informed of patients receiving daratumumab to allow for the identification of safe blood products in these patients. Baseline compatibility typing should be performed before daratumumab treatment is started. Results should be recorded and referenced for all transfusions. For emergency transfusions, non-cross-matched, ABO-RhDcompatible RBCs can be given.

\section{Elotuzumab}

Elotuzumab (ELO) is an IV monoclonal antibody which targets the myeloma cells expressing signaling lymphocyte activation family 7 (SLAMF-7), which leads to cell death through promoting natural killer-mediated myeloma cell death. ${ }^{43,85}$ In a Phase III study, 321 patients were assigned to receive ELO and $\mathrm{Rd}$, with 325 patients assigned to receive $\mathrm{Rd}$ alone. At 1 year, there was an improved PFS in the ELO and Rd group versus $\mathrm{Rd}$ alone ( $68 \%$ vs $57 \%$ ), and at 2 years, the rates were $41 \%$ and $27 \%$, respectively. The ORR in the ELO group was $79 \%$ versus $66 \%$ in the $\mathrm{Rd}$ group $(P<0.001)$. A $30 \%$ relative reduction in $\mathrm{PD}$ or death was observed. Common grade 3 or 4 AEs in the two groups were lymphocytopenia, neutropenia, fatigue, and pneumonia. Infusion reactions were generally mild, but occurred in 33 patients (10\%) in the ELO group and were grade 1 or 2 in 29 patients. Based on these side effects, regular complete blood count monitoring and premedication of ELO with corticosteroids is recommended.

\section{Response monitoring and clonal evolution}

Most patients will achieve a remission and then ultimately relapse. Remissions and relapses can occur over time and lead to clonal evolution, drug resistance, and ultimately drug-resistant disease. ${ }^{86-88}$ Close monitoring of response to 
therapy and evaluation for biochemical and symptomatic disease progression are vital to prevent disease-related complications.

\section{Supportive care}

Patients are living longer than ever, in part, due to advances in supportive care within the last 20 years. Attention must be given to prevent and intervene on common myeloma-related complications. These include prevention of skeletal-related events with bisphosphonates, avoidance of nephrotoxic agents such as nonsteroidal anti-inflammatory agents or contrast dyes, immunization, and use of appropriate antiinfective and antiviral agents to treat infections.

\section{Bone}

Bone complications are common at diagnosis and throughout. Multiple cytokines and mechanisms are responsible for bone loss in MM, yet an age- or disease-related imbalance in osteoblast and osteoclast system occurs. Accelerated osteoclast activity without osteoblastic stimulation leads to bone turnover. ${ }^{13,14,89,90}$ Therefore, all patients with MM should receive bisphosphonates on a monthly basis for at least 12 months to prevent skeletalrelated events, and possible antimyeloma benefit. ${ }^{14,91-95} \mathrm{~A}$ baseline dental evaluation and ongoing surveillance of complications such as osteonecrosis of the jaw and renal toxicity should be employed. ${ }^{96}$ Regular physical activity such as walking, swimming, or light weight-bearing exercise should be encouraged to maintain mobility, decrease the risk of falls, and for overall health. ${ }^{97}$ Regular monitoring of vitamin D levels and routine calcium and vitamin D intake are also recommended. ${ }^{96,98}$

\section{Renal}

The etiology of renal impairment in patients with MGUS or MM can be due to the disease itself or multifactorial. Patients with older age, long-standing comorbid illnesses such as hypertension or diabetes, hydronephrosis, renal obstruction, acute tubular injury, or concurrent amyloidosis are at a higher risk for renal insufficiency. ${ }^{99}$ In patients with active MM, excess urinary light chains can overwhelm the proximal tubules as the level of light chain burden increases, hindering the kidneys' ability to compensate, and combine with Tamm-Horsfall mucoprotein at the level of the nephrons, leading to cast nephropathy. ${ }^{99}$

As a result of these MM-related and other factors, $\sim 50 \%$ of patients with MM will experience renal insufficiency throughout the course of their disease. ${ }^{99}$ Medications such as nonsteroidal anti-inflammatory drugs, IV dyes, aminoglycoside antibiotics, and loop diuretics should thus be avoided, unless patients are closely monitored. Avoidance of dehydration with adequate fluid intake, especially if patients are experiencing diarrhea, which can occur with bortezomib, panobinostat, or long-term lenalidomide use, is recommended. ${ }^{100}$ In patients with renal failure, aggressive oral or IV hydration and treatment of the disease or underlying cause is warranted.

\section{Infections}

Infections remain a common cause of early MM mortality, whether from neutropenia secondary to chemotherapy or from the disease itself. Bacterial and/or viral infections are common in MM and a leading cause of death. ${ }^{101,102}$ Functional impaired immunoglobulin synthesis, altered antibody formation after antigenic stimulation, and treatment-related myelosuppression from chemotherapy or steroid therapy place patients at risk to develop life-threatening infections.

The most common types of infections at diagnosis are Streptococcus pneumoniae, Haemophilus influenzae, and herpes shingles virus, although other types have been reported. ${ }^{103}$ Seasonal inactivated influenza vaccination and immunization with polyvalent pneumococcal vaccines every 5 years (pneumococcal conjugate vaccine-13 and pneumococcal polysaccharide vaccine 23) are recommend for all patients. ${ }^{104}$ Attention to common causative organisms and appropriate and prompt antibiotic selection to treat these organisms are essential. For life-threatening or recurrent infections, IV immunoglobulin (IVIg) should be considered. ${ }^{33}$

\section{Peripheral neuropathy}

Peripheral neuropathy can occur at diagnosis in a majority of individuals (20\%), and in up to $75 \%$, it occurs throughout the course of one's illness. Improved survival and a longer lifespan for patients with MM are counterbalanced by these common and deleterious side effects of treatment. Peripheral neuropathy is commonly cited as a main side effect of MM therapy that affects one's quality of life in a negative fashion. ${ }^{105-107}$ Moderate-to-severe peripheral neuropathy secondary to bortezomib generally occurs within 4-6 months and can limit one's ability to receive effective antimyeloma therapy with new and yet undiscovered agents. ${ }^{108}$ Ixazomib, carfilzomib, vincristine, and thalidomide are agents which can cause neurotoxicity to a lesser extent than bortezomib. ${ }^{109}$

No gold standard for the diagnosis of peripheral neuropathy exists. Electromyography, nerve conduction studies, and quantitative sensory testing are methods to quantify 
the extent of muscular or sensory nerve impairment. These techniques can be painful and often fail to capture the extent of the symptoms. ${ }^{110,111}$ Patients' self-report of numbness, muscle weakness, tingling or paresthesias, combined with an astute physical examination are the two most reliable methods to report new-onset or worsening symptoms. ${ }^{112}$ Since self-report is of high importance, the patient and caregiver must be educated on signs and symptoms of peripheral neuropathy, and advised to alert the providers of these symptoms. Prompt intervention of symptoms with holding, dose reduction, or discontinuation of the offending agent can lead to improvement of neuropathy symptoms. ${ }^{106}$ Investigation into secondary causes of neuropathy, such as vitamin B6, B12 deficiencies, should be explored, as vitamin B deficiencies and diabetes are fairly common in MM. ${ }^{106,113,114}$

\section{Deep vein thrombosis (DVT) andVTEs}

VTEs encompass DVT, which occurs in an extremity, and pulmonary embolism (PE). Patients with MM are at risk for life-threatening VTEs, as a result of their disease as well as individual and inherited risk factors. Immunomodulatory drugs, anthracyclines, carfilzomib (at a dose of $56 \mathrm{mg} / \mathrm{m}^{2} \mathrm{IV}$ twice weekly), and erythropoiesis stimulating agents further contribute to the risk. ${ }^{115}$ For all patients on IMiDs and carfilzomib, VTE risk stratification is imperative. Factors such as surgery, previous DVT or PE, obesity, history of cardiac or renal disease, and combination chemotherapy should be considered. If patients have nil or one risk factor, aspirin is recommended to further reduce the risk. ${ }^{42,59,115,116}$

Ongoing patient education should be targeted toward signs and symptoms of DVT or PE with prompt reporting of unilateral edema and coolness or pain in an extremity. Severe symptoms of PE with acute shortness of breath, anxiety, and tachycardia require emergent management. Ambulation and adequate hydration is recommended for all patients. Prophylactic anticoagulation with low-molecular-weight heparin in patients while being hospitalized, or full anticoagulation with warfarin, enoxaparin, or direct oral anticoagulants (in patients with glomerular filtration rate $>30$ ) in patients at a high risk for thrombosis is recommended. ${ }^{117,118}$

\section{Survivorship in MM}

Survivorship was once considered as the time period after the individual successfully completed cancer treatment. Since treatment is often ongoing in MM, survivorship in MM begins at diagnosis and continues through the balance of the individual's life. ${ }^{119}$ The importance of survivorship in cancer and the generation of a survivorship care plan (SCP) emerged following the release of an Institute of Medicine report that focused on the importance of health prevention and promotion models of care for cancer survivors. ${ }^{120}$ To guide patients and primary providers on personalized screening recommendations based on prior chemotherapy, age, and other risk factors, an SCP is recommended for each individual with cancer. The provision of a care plan should be twofold and include: 1) a treatment summary and 2) essential components of a healthy lifestyle which include coordination of care among primary and other providers, health maintenance recommendations, early detection and screening, and psychosocial welfare. ${ }^{119,121}$

Until the last decade, patients with MM were not considered cancer survivors due to lack of effective treatment options. Patients with MM are living longer than ever, and health promotion and disease prevention are as important among this group as in others with a chronic illness. ${ }^{122}$ Due to the complexity of current regimens to treat MM, a multidisciplinary team of physicians, advanced practice providers (such as nurse practitioners or physician assistants), nurses, pharmacists, and social workers is essential to enhance outcomes. Each member can take on a different role to educate patients on the complex treatment regimens, review and recommend supportive therapies (such as bisphosphonates, immunizations, and blood transfusions), and attend to symptom management (constipation, diarrhea, and other complications). Clinicians should consider the use of a treatment summary and/or provide an SCP to each patient to promote one's understanding of the disease and enhance adherence. ${ }^{121}$ The SCP can be as simple as a calendar list of appointments, printout, and review of current medications with recommended dose, duration, and rationale, and assessment and intervention of side effects. The importance of health maintenance with routine cancer and cardiovascular screening and partnership with a primary care provider should be emphasized for all.

\section{Conclusion}

MM is a heterogeneous disorder of the plasma cell, which remains incurable, but highly treatable. Advances in the understanding of plasma cell development within the bone marrow milieu have led to newer therapies with sophisticated mechanisms of action. It is essential for clinicians to be aware of the new drugs and common side effects for effective patient management. The provision of care plans, calendars, or other tools can help patients understand and adhere to treatment, and allow the patient to participate in one's care. 


\section{Acknowledgments}

BF serves as a consultant for Amgen, Celgene, and Takeda. JV is in speakers bureau of Celgene, Takeda, and Amgen.

\section{Disclosure}

The authors report no conflicts of interest in this work.

\section{References}

1. Kyle RA, Gertz MA, Witzig TE, et al. Review of 1027 patients with newly diagnosed multiple myeloma. Mayo Clin Proc. 2003;78(1): 21-33.

2. Cancer IAfRo. GLOBOCAN 2012: Estimated Cancer Incidence, Mortality and Prevalence Worldwide. Available from: http://globocan.iarc. fr/Pages/fact_sheets_population.aspx?country=900. Accessed April 13, 2016.

3. Landgren O, Rajkumar SV, Pfeiffer RM, et al. Obesity is associated with an increased risk of monoclonal gammopathy of undetermined significance among black and white women. Blood. 2010; 116(7):1056-1059.

4. Rajkumar SV, Dimopoulos MA, Palumbo A, et al. International Myeloma Working Group updated criteria for the diagnosis of multiple myeloma. Lancet Oncol. 2014;15(12):e538-e548.

5. Faiman B. Myeloma genetics and genomics: practice implications and future directions. Clin Lymphoma Myeloma Leuk. 2014; 14(6):436-440.

6. Fonseca R, Monge J. Myeloma: Classification and risk assessment. Semin Oncol. 2013;40(5):554-566.

7. Landgren O, Morgan GJ. Biological frontiers in multiple myeloma: from biomarker identification to clinical practice. Clin Cancer Res. Epub 2013 Nov 22

8. Van Wier S, Braggio E, Baker A, et al. Hypodiploid multiple myeloma is characterized by more aggressive molecular markers than nonhyperdiploid multiple myeloma. Haematologica. Epub 2013 May 28.

9. Vincent Rajkumar S. Multiple myeloma: 2014 Update on diagnosis, risk-stratification, and management. Am J Hematol. 2014;89(10): 999-1009.

10. Shah JJ, Abonour R, Durie BGM, et al. Connect $\mathrm{MM}^{\circledR}$-the multiple myeloma (MM) disease registry: interim analysis of overall survival and outcomes in patients with high-risk disease. Blood. 2014; 124(21):2106

11. Terpos E, Moulopoulos LA, Dimopoulos MA. Advances in imaging and the management of myeloma bone disease. J Clin Oncol. 2011; 29(14):1907-1915.

12. Roodman GD. Mechanisms of bone metastasis. $N$ Engl J Med. 2004;350(16):1655-1664.

13. Raje N, Roodman GD. Advances in the biology and treatment of bone disease in multiple myeloma. Clin Cancer Res. 2011;17(6): 1278-1286.

14. Terpos E, Morgan G, Dimopoulos MA, et al. International Myeloma Working Group recommendations for the treatment of multiple myelomarelated bone disease. J Clin Oncol. 2013;31(18):2347-2357.

15. Dimopoulos MA, Hillengass J, Usmani S, et al. Role of magnetic resonance imaging in the management of patients with multiple myeloma: a consensus statement. J Clin Oncol. 2015;33(6):657-664.

16. Durie BGM, Harousseau JL, Miguel JS, et al. International uniform response criteria for multiple myeloma. Leukemia. 2006;20(9): 1467-1473.

17. Landgren O, Graubard BI, Katzmann JA, et al. Racial disparities in the prevalence of monoclonal gammopathies: a population-based study of 12,482 persons from the National Health and Nutritional Examination Survey. Leukemia. 2014;28(7):1537-1542.

18. Rajkumar SV, Kyle RA, Therneau TM, et al. Serum free light chain ratio is an independent risk factor for progression in monoclonal gammopathy of undetermined significance. Blood. 2005;106(3):812-817.
19. Katzmann JA, Clark R, Kyle RA, et al. Suppression of uninvolved immunoglobulins defined by heavy/light chain pair suppression is a risk factor for progression of MGUS. Leukemia. 2013;27(1):208-212.

20. Kyle RA, Durie BGM, Rajkumar SV, et al. Monoclonal gammopathy of undetermined significance (MGUS) and smoldering (asymptomatic) multiple myeloma: IMWG consensus perspectives risk factors for progression and guidelines for monitoring and management. Leukemia. 2010;24(6):1121-1127.

21. Larsen JT, Kumar SK, Dispenzieri A, Kyle RA, Katzmann JA, Rajkumar SV. Serum free light chain ratio as a biomarker for highrisk smoldering multiple myeloma. Leukemia. 2013;27(4):941-946.

22. Rajkumar SV. Evolving diagnostic criteria for multiple myeloma. Hematology Am Soc Hematol Educ Program. 2015;2015(1):272-278.

23. Waxman A, Mick R, Garfall A, et al. Modeling the risk of progression in smoldering multiple myeloma. J Clin Oncol. 2014;32:5s,(suppl):abstract 8607.

24. Dispenzieri A, Kyle R, Merlini G, et al. International Myeloma Working Group guidelines for serum-free light chain analysis in multiple myeloma and related disorders. Leukemia. 2008;23(2):215-224.

25. Dispenzieri A, Kyle RA, Katzmann JA, et al. Immunoglobulin free light chain ratio is an independent risk factor for progression of smoldering (asymptomatic) multiple myeloma. Blood. 2008;111(2):785-789.

26. Kastritis E, Terpos E, Moulopoulos L, et al. Extensive bone marrow infiltration and abnormal free light chain ratio identifies patients with asymptomatic myeloma at high risk for progression to symptomatic disease. Leukemia. 2013;27(4):947-953.

27. Hillengass J, Weber MA, Kilk K, et al. Prognostic significance of wholebody MRI in patients with monoclonal gammopathy of undetermined significance. Leukemia. 2014;28(1):174-178.

28. Durie BG, Salmon SE. A clinical staging system for multiple myeloma: correlation of measured myeloma cell mass with presenting clinical features, response to treatment and survival. Cancer. 1975;36: 842-854.

29. Greipp PR, Miguel JS, Durie BGM, et al. International staging system for multiple myeloma. J Clin Oncol. 2005;23(15):3412-3420.

30. Lonial S, Anderson KC. Association of response endpoints with survival outcomes in multiple myeloma. Leukemia. 2014;28(2):258-268.

31. Fonseca R, Barlogie B, Bataille R, et al. Genetics and cytogenetics of multiple myeloma. Cancer Res. 2004;64(4):1546-1558.

32. Palumbo A, Avet-Loiseau H, Oliva S, et al. Revised international staging system for multiple myeloma: a report from International Myeloma Working Group. J Clin Oncol. Epub 2015 Aug 3.

33. NCCN. NCCN Multiple Myeloma Clinical Practice Guidelines in Oncology v2.2016. The Complete Library of NCCN Clinical Practice Guidelines in Oncology. Jenkintown, PA: Author; 2016.

34. Kumar SK, Rajkumar SV, Dispenzieri A, et al. Improved survival in multiple myeloma and the impact of novel therapies. Blood. 2008; 111(5):2516-2520.

35. Richardson PG, Barlogie B, Berenson J, et al. A phase 2 study of bortezomib in relapsed, refractory myeloma. $N$ Engl J Med. 2003; 348(26):2609-2617.

36. Richardson PG, Sonneveld P, Schuster MW, et al; Assessment of Proteasome Inhibition for Extending Remissions (APEX) Investigators. Bortezomib or high-dose dexamethasone for relapsed multiple myeloma. N Engl J Med. 2005;352(24):2487-2498.

37. Richardson PG, Blood E, Mitsiades CS, et al. A randomized phase 2 study of lenalidomide therapy for patients with relapsed or relapsed and refractory multiple myeloma. Blood. 2006;108(10):3458-3464.

38. Richardson PG, Sonneveld P, Schuster M, et al. Extended follow-up of a phase 3 trial in relapsed multiple myeloma: final time-to-event results of the APEX trial. Blood. 2007;110(10):3557-3560.

39. San Miguel JF, Schlag R, Khuageva NK, et al; VISTA Trial Investigators. Bortezomib plus melphalan and prednisone for initial treatment of multiple myeloma. N Engl J Med. 2008;359(9):906-917.

40. Richardson P, Jagannath S, Hussein M, et al. Safety and efficacy of single-agent lenalidomide in patients with relapsed and refractory multiple myeloma. Blood. 2009;114(4):772-778. 
41. Richardson PG, Schlossman RL, Alsina M, et al. PANORAMA 2: panobinostat in combination with bortezomib and dexamethasone in patients with relapsed and bortezomib-refractory myeloma. Blood. 2013;122(14):2331-2337.

42. Richardson PG, Siegel DS, Vij R, et al. Pomalidomide alone or in combination with low-dose dexamethasone in relapsed and refractory multiple myeloma: a randomized phase 2 study. Blood. 2014;123(12): 1826-1832.

43. Lonial S, Dimopoulos M, Palumbo A, et al; ELOQUENT-2 Investigators. Elotuzumab therapy for relapsed or refractory multiple myeloma. N Engl J Med. 2015;373(7):621-631.

44. Stewart AK, Rajkumar SV, Dimopoulos MA, et al; ASPIRE Investigators. Carfilzomib, lenalidomide, and dexamethasone for relapsed multiple myeloma. N Engl J Med. 2015;372(2):142-152.

45. Benboubker L, Dimopoulos MA, Dispenzieri A, et al; FIRST Trial Team. Lenalidomide and dexamethasone in transplant-ineligible patients with myeloma. N Engl J Med. 2014;371(10):906-917.

46. Richardson PG, Jagannath S, Moreau P, et al. Final results for the 1703 phase $1 \mathrm{~b} / 2$ study of elotuzumab in combination with lenalidomide and dexamethasone in patients with relapsed/refractory multiple myeloma. In: 56th ASH Annual Meeting and Exposition; December 6-9, 2014; San Francisco, CA; Vol 1242014.

47. Richardson P, Weller E, Lonial S, et al. Lenalidomide, bortezomib, and dexamethasone combination therapy in patients with newly diagnosed multiple myeloma [abstract 92]. Blood. 2010;116(5):679-686.

48. Dimopoulos MA, Kastritis E, Christoulas D, et al. Treatment of patients with relapsed/refractory multiple myeloma with lenalidomide and dexamethasone with or without bortezomib: prospective evaluation of the impact of cytogenetic abnormalities and of previous therapies. Leukemia. 2010;24(10):1769-1778.

49. Durie B, Hoering A, Rajkumar SV, et al. Bortezomib, lenalidomide and dexamethasone vs. lenalidomide and dexamethasone in patients (Pts) with previously untreated multiple myeloma without an intent for immediate autologous stem cell transplant (ASCT): results of the randomized phase III trial SWOG S0777. Blood. 2015;126(23):25.

50. Moreau P, Pylypenko H, Grosicki S, et al. Subcutaneous versus intravenous administration of bortezomib in patients with relapsed multiple myeloma: a randomised, phase 3, non-inferiority study. Lancet Oncol 2011;12(5):431-440.

51. Faiman B, Elson P, Smith M, et al. Neuropathy and efficacy of weekly subcutaneous bortezomib in myeloma and AL amyloidosis. Blood. 2013;122(21):1975.

52. Rajkumar SV, Jacobus S, Callander NS, et al; Eastern Cooperative Oncology Group. Lenalidomide plus high-dose dexamethasone versus lenalidomide plus low-dose dexamethasone as initial therapy for newly diagnosed multiple myeloma: an open-label randomised controlled trial. Lancet Oncol. 2010;11(1):29-37.

53. Weber DM, Chen C, Niesvizky R, et al; Multiple Myeloma (009) Study Investigators. Lenalidomide plus dexamethasone for relapsed multiple myeloma in North America. $N$ Engl J Med. 2007; 357(21):2133-2142.

54. Wang M, Dimopoulos MA, Chen C, et al. Lenalidomide plus dexamethasone is more effective than dexamethasone alone in patients with relapsed or refractory multiple myeloma regardless of prior thalidomide exposure. Blood. 2008;112(12):4445-4451.

55. Palumbo A, Rajkumar SV, San Miguel JF, et al. International Myeloma Working Group consensus statement for the management, treatment, and supportive care of patients with myeloma not eligible for standard autologous stem-cell transplantation. J Clin Oncol. 2014;32(6):587-600.

56. Larocca A, Palumbo A. How I treat fragile myeloma patients. Blood. Epub 2015 Aug 31.

57. Chen N, Lau H, Kong L, et al. Pharmacokinetics of lenalidomide in subjects with various degrees of renal impairment and in subjects on hemodialysis. J Clin Pharmacol. 2007;47(12):1466-1475.

58. Palumbo A, Bringhen S, Ludwig H, et al. Personalized therapy in multiple myeloma according to patient age and vulnerability: a report of the European Myeloma Network (EMN). Blood. 2011;118(17):4519-4529.
59. Dimopoulos MA, Moreau P, Palumbo A, et al; ENDEAVOR investigators. Carfilzomib and dexamethasone versus bortezomib and dexamethasone for patients with relapsed or refractory multiple myeloma (ENDEAVOR): a randomised, phase 3, open-label, multicentre study. Lancet Oncol. 2016;17(1):27-38.

60. Siegel D, Martin T, Wang M, et al. A phase 2 study of single-agent carfilzomib (PX-171-003-A1) in patients with relapsed and refractory multiple myeloma. Blood. 2012;120(14):2817-2825.

61. Jagannath S, Vij R, Stewart AK, et al. An open-label single-arm pilot phase II study (PX-171-003-A0) of low-dose, single-agent carfilzomib in patients with relapsed and refractory multiple myeloma. Clin Lymphoma Myeloma Leuk. 2012;12(5):310-318.

62. Vij R, Siegel D, Jagannath S, et al. An open-label, single-arm, phase 2 study of single-agent carfilzomib in patients with relapsed and/or refractory multiple myeloma who have been previously treated with bortezomib. Br J Haematol. 2012;158(6):739-748.

63. Vij R, Wang M, Kaufman JL, et al. An open-label, single-arm, phase 2 (PX-171-004) study of single-agent carfilzomib in bortezomib-naive patients with relapsed and/or refractory multiple myeloma. Blood. 2012;119(24):5661-5670.

64. Badros AZ, Vij R, Martin T, et al. Carfilzomib in multiple myeloma patients with renal impairment: pharmacokinetics and safety. Leukemia. Epub 2013 Jan 31.

65. Palumbo A, Rossi D, Bringhen S, et al. Weekly carfilzomib, cyclophosphamide and dexamethasone (wCCd) in newly diagnosed multiple myeloma patients: A phase I-II study. In: 56th ASH Annual Meeting and Exposition; December 6-9, 2014; San Francisco, CA; Vol. 1242014.

66. Squifflet P, Michiels S, Siegel DS, Vij R, Ro S, Buyse ME. Multivariate modelling reveals evidence of a dose-response relationship in phase 2 studies of single-agent carfilzomib [abstract]. Blood. 2011;118(21):Abstract 1877.

67. Lendvai N, Landau H, Lesokhin A, et al. Phase II study of infusional carfilzomib in patients with relapsed or refractory multiple myeloma. Blood. 2012;120:Abstract 947.

68. Stadtmauer E, Shah J, Abonour R, et al. Carfilzomib, pomalidomide and dexamethasone (CPomd) for relapsed/refractory multiple myeloma (RRMM): a phase I/II trial. Clin Lymphoma Myeloma Leuk. 2013;13(Supplement 1):P250.

69. Shah J, Thomas S, Weber D, et al. Phase $1 / 1$ b study of the efficacy and safety of the combination of panobinostat + carfilzomib in patients with relapsed and/or refractory multiple myeloma. Blood. 2012;120:Abstract 4081.

70. Berdeja J, Hart L, Lamar R, Murphy P, Morgan S, IFlinn I. Phase I/II study of panobinostat and carfilzomib in patients (pts) with relapsed or refractory multiple myeloma (MM), interim phase I safety analysis. Blood. 2012;120:Abstract 4048.

71. Kaufman J, Zimmerman T, Jakubowiak A, et al. Phase I study of the combination of carfilzomib and panobinostat for patients with relapsed and refractory myeloma: a multicenter MMRC clinical trial. Haematologica. 2013;98(Supplement 1):Abstract P771.

72. Amgen. KYPROLIS ${ }^{\text {TM }}$ (carfilzomib) for Injection, for intravenous use South San Francisco, CA; 2016.

73. Colson K. Treatment-related symptom management in patients with multiple myeloma: a review. Supportive Care Cancer. 2015;23(5): 1431-1445.

74. Corporation C. Pomalidomide (Pomalyst) [package insert]. Summit, NJ: Author; 2013.

75. Lacy MQ, LaPlant BR, Laumann KM, et al. Pomalidomide, bortezomib and dexamethasone (PVD) for patients with relapsed lenalidomide refractory multiple myeloma (MM). In: 56th ASH Annual Meeting and Exposition; December 6-9, 2014; San Francisco, CA; Vol 1242014.

76. Baz R, Martin TG, Alsina M, et al. Pomalidomide, cyclophosphamide, and dexamethasone is superior to pomalidomide and dexamethasone in relapsed and refractory myeloma: results of a multicenter randomized phase II study. In: 56th ASH Annual Meeting and Exposition; December 6-9, 2014; San Francisco, CA; Vol. 1242014. 
77. Badros AZ, Kocoglu MH, Ma N, et al. A phase II study of anti PD-1 antibody pembrolizumab, pomalidomide and dexamethasone in patients with relapsed/refractory multiple myeloma (RRMM). Blood. 2015;126(23):506.

78. Chari A, Lonial S, Suvannasankha A, et al. Open-label, multicenter, phase $1 \mathrm{~b}$ study of daratumumab in combination with pomalidomide and dexamethasone in patients with at least 2 lines of prior therapy and relapsed or relapsed and refractory multiple myeloma. Blood. 2015;126(23):508.

79. San-Miguel JF, Hungria VT, Yoon SS, et al. Panobinostat plus bortezomib and dexamethasone versus placebo plus bortezomib and dexamethasone in patients with relapsed or relapsed and refractory multiple myeloma: a multicentre, randomised, double-blind phase 3 trial. Lancet Oncol. 2014;15(11):1195-1206.

80. Richardson PG, Hungria VT, Yoon SS, et al. Panobinostat plus bortezomib and dexamethasone in relapsed/relapsed and refractory myeloma: outcomes by prior treatment. Blood. Epub 2015 Dec 2.

81. Moreau P, Masszi T, Grzasko N, et al. Ixazomib, an investigational oral proteasome inhibitor $(\mathrm{PI})$, in combination with lenalidomide and dexamethasone (IRd), significantly extends progression-free survival (PFS) for patients (Pts) with relapsed and/or refractory multiple myeloma (RRMM): the phase 3 tourmaline-MM1 study (NCT01564537). Blood. 2015;126(23):727.

82. Usmani S, Weiss B, Bahlis NJ, et al. Clinical efficacy of daratumumab monotherapy in patients with heavily pretreated relapsed or refractory multiple myeloma. Blood. 2015;126(23):29.

83. Plesner T, Arkenau H-T, Gimsing P, et al. Daratumumab in combination with lenalidomide and dexamethasone in patients with relapsed or relapsed and refractory multiple myeloma: updated results of a phase 1/2 study (GEN503). Blood. 2015;126(23):507.

84. Janssen. Daratumumab (DARZALEX) injection. 2015.

85. Hsi ED, Steinle R, Balasa B, et al. CS1, a potential new therapeutic antibody target for the treatment of multiple myeloma. Clin Cancer Res. 2008;14(9):2775-2784.

86. Egan JB, Shi C-X, Tembe W, et al. Whole-genome sequencing of multiple myeloma from diagnosis to plasma cell leukemia reveals genomic initiating events, evolution, and clonal tides. Blood. 2012; 120(5):1060-1066.

87. Keats JJ, Chesi M, Egan JB, et al. Clonal competition with alternating dominance in multiple myeloma. Blood. 2012;120(5):1067-1076.

88. Lohr Jens G, Stojanov P, Carter Scott L, et al. Widespread genetic heterogeneity in multiple myeloma: implications for targeted therapy. Cancer Cell. 2014;25(1):91-101.

89. Raje NS, Willenbacher W, Hungria V, et al. Evaluating results from the multiple myeloma subset of patients treated with denosumab or zoledronic acid (ZA) in a randomized phase III study. ASCO Meeting Abstracts. 2013;31(15_suppl):8589.

90. Terpos E, Berenson J, Raje N, Roodman GD. Management of bone disease in multiple myeloma. Expert Rev Hematol. 2014;7(1):113-125.

91. Berenson JR. Efficacy of pamidronate in reducing skeletal events in patients with advanced multiple myeloma. Myeloma Aredia Study Group. N Engl J Med. 1996;334:488-493.

92. Machado M, Cruz LS, Tannus G, Fonseca M. Efficacy of clodronate, pamidronate, and zoledronate in reducing morbidity and mortality in cancer patients with bone metastasis: a meta-analysis of randomized clinical trials. Clin Ther. 2009;31(5):962-979.

93. Aapro M, Saad F, Costa L. Optimizing clinical benefits of bisphosphonates in cancer patients with bone metastases. Oncologist. 2010; 15(11):1147-1158.

94. Gimsing P, Carlson K, Turesson I, et al. Effect of pamidronate $30 \mathrm{mg}$ versus $90 \mathrm{mg}$ on physical function in patients with newly diagnosed multiple myeloma (Nordic Myeloma Study Group): a double-blind, randomised controlled trial. Lancet Oncol. 2010;11(10):973-982.

95. Morgan GJ, Davies FE, Gregory WM, et al; National Cancer Research Institute Haematological Oncology Clinical Study Group. First-line treatment with zoledronic acid as compared with clodronic acid in multiple myeloma (MRC Myeloma IX): a randomised controlled trial. Lancet. 2010;376(9757):1989-1999.
96. Miceli T, Colson K, Faiman B, Miller K, Tariman J; International Myeloma Foundation Nurse Leadership Board. Maintaining bone health in patients with multiple myeloma. Clin J Oncol Nurs. 2011;15:9-23.

97. Rome SI, Jenkins BS, Lilleby KE; International Myeloma Foundation Nurse Leadership Board. Mobility and safety in the multiple myeloma survivor: survivorship care plan of the International Myeloma Foundation Nurse Leadership Board. Clin J Oncol Nurs. 2011;15 (Suppl):41-52.

98. Bilotti E, Faiman B, Richards T, Tariman JD, Miceli T, Rome S; International Myeloma Foundation Nurse Leadership Board. Survivorship care guidelines for patients living with multiple myeloma: consensus statements of the International Myeloma Foundation Nurse Leadership Board. Clin J Oncol Nurs. 2011;15(Supp1):5-8.

99. Faiman B, Mangan P, Spong JE, Tariman JD; The International Myeloma Foundation Nurse Leadership Board. Renal complications in multiple myeloma and related disorders: survivorship care plan of the IMF Nurse Leadership Board. Clin J Oncol Nurs. 2011;15 (Suppl):66-76.

100. Faiman B, Sidana S, Elson P, et al. Lenalidomide related diarrhea correlates with survival in multiple myeloma. Blood. 2013;122(21): 5397.

101. Nucci M, Anaissie E. Infections in patients with multiple myeloma in the era of high-dose therapy and novel agents. Clin Infect Dis. 2009;49(8):1211-1225.

102. Kalambokis GN, Christou L, Tsianos EV. Multiple myeloma presenting with an acute bacterial infection. Int J Lab Hematol. 2009;31(4):375-383.

103. Durie BG, Kyle RA, Belch A, et al; Scientific Advisors of the International Myeloma Foundation. Myeloma management guidelines: a consensus report from the Scientific Advisors of the International Myeloma Foundation. Hematol J. 2003;4(6):379-398.

104. CDC. Prevention and control of influenza with vaccines. Recommendations of the Advisory Committee on Immunization Practices (ACIP). MMWR. 2010;59(8)

105. Visovsky C, Haas M, Faiman B, et al. Nurse self-evaluation of assessment of chemotherapy-induced peripheral neuropathy in patients with cancer. $J$ Adv Pract Oncol. 2012;3:319-325.

106. Richardson PG, Delforge M, Beksac M, et al. Management of treatment-emergent peripheral neuropathy in multiple myeloma. Leukemia. 2012;26(4):595-608.

107. Tariman J, Love G, McCullagh E, Sandifer S; IMF Nurse Leadership Board. Peripheral neuropathy associated with novel therapies in patients with multiple myeloma: consensus statement of the IMF nurse leadership board. Clin J Oncol Nurs. 2008;12:29-35.

108. Broyl A, Jongen JLM, Sonneveld P. General aspects and mechanisms of peripheral neuropathy associated with bortezomib in patients with newly diagnosed multiple myeloma. Semin Hematol. 2012; 49(3):249-257.

109. Oncology T. Bortezomib (Velcade) [package insert]. Cambridge, MA: Author; 2015.

110. Preston D, Shapiro B, editors. Electromyography and Neuromuscular Disorders: Clinical-Electrophysiologic Correlations. 2 ed. Boston: Butterworth-Heinemann; 2005.

111. Poncelet AN. An algorithm for the evaluation of peripheral neuropathy. Am Fam Physician. 1998;15(57):755-764.

112. Calhoun E, Welshman E, Chang C, et al. Psychometric Evaluation of the Functional Assessment of Cancer Therapy/Gynecologic Oncology Group-Neurotoxicity (FACT/GOG-Ntx) questionnaire for patients receiving systemic chemotherapy. Int $J$ Gynecol Cancer. 2003;13:741-748.

113. Baz R, Alemany C, Green R, Hussein MA. Prevalence of vitamin B12 deficiency in patients with plasma cell dyscrasias. Cancer. 2004;101(4):790-795.

114. Badros A, Goloubeva O, Dalal JS, et al. Neurotoxicity of bortezomib therapy in multiple myeloma: a single-center experience and review of the literature. Cancer. 2007;110(5):1042-1049.

115. Palumbo A, Rajkumar SV, Dimopoulos MA, et al; International Myeloma Working Group. Prevention of thalidomide- and lenalidomideassociated thrombosis in myeloma. Leukemia. 2008;22(2):414-423. 
116. Baz R, Li L, Kottke-Marchant K, et al. The role of aspirin in the prevention of thrombotic complications of thalidomide and anthracycline-based chemotherapy for multiple myeloma. Mayo Clin Proc. 2005;80(12):1568-1574.

117. Lyman GH, Bohlke K, Khorana AA, et al. Venous thromboembolism prophylaxis and treatment in patients with cancer: American Society of Clinical Oncology clinical practice guideline update 2014. J Clin Oncol. 2015;33(6):654-656.

118. van Es N, Büller HR. Using direct oral anticoagulants (DOACs) in cancer and other high-risk populations. Hematology Am Soc Hematol Educ Program. 2015;2015(1):125-131.

119. Denlinger CS, Carlson RW, Are M, et al. Survivorship: introduction and definition. Clinical practice guidelines in oncology. J Natl Compr Canc Netw. 2014;12(1):34-45.

120. National Cancer Institute. About cancer survivorship research: survivorship definitions. 2014; Available from: http://cancercontrol.cancer. gov/ocs/. Accessed August 22, 2014.

121. Taylor K, Monterosso L. Survivorship care plans and treatment summaries in adult patients with hematologic cancer: an integrative literature review. Oncol Nurs Forum. 2015;42(3):283-291.
122. Bilotti E, Gleason C, McNeill A; International Myeloma Foundation Nurse Leadership Board. Routine health maintenance in patients living with multiple myeloma: survivorship care plan of the International Myeloma Foundation Nurse Leadership Board. Clin J Oncol Nurs. 2011;15 Supp:25-40.

123. Faiman B, Bilotti E, Mangan P, Rogers K. Steroid-associated side effects in patients with multiple myeloma: consensus statement of the IMF Nurse Leadership Board. Clinical Journal of Oncology Nursing. 2008; 12:53-62.

124. Rome S, Doss D, Miller K, Westphal J. Thromboembolic events associated with novel therapies in patients with multiple myeloma: Consensus statement of the IMF Nurse Leadership Board. Clinical Journal of Oncology Nursing. 2008;12:21-28.

125. Richards T and Brigle KJ. Palliative care in multiple myeloma. $J A d v$ Pract Oncol. 2016;7:31-43.

\section{Publish your work in this journal}

Blood and Lymphatic Cancer: Targets and Therapy is an international, peer-reviewed, open access journal focusing on blood and lymphatic cancer research, identification of therapeutic targets and the optimal use of preventative and integrated treatment interventions to achieve improved outcomes, enhanced survival and quality of life for the cancer patient. The manuscript management system is completely online and includes a very quick and fair peer-review system. Visit http://www.dovepress.com/testimonials.php to read real quotes from published authors.

Submit your manuscript here: http://www.dovepress.com/blood-and-lymphatic-cancer-targets-and-therapy-journal 